\title{
Orbital Trends of Binary Pulsars
}

\author{
Tapan Chatterjee \\ University of Puebla (FCC), Apartado Postal 1316, Puebla, Mexico
}

\begin{abstract}
We try to understand the eccentricity of high-mass binary pulsars using some methods of statistical mechanics.
\end{abstract}

\section{Introduction}

There is a high abundance of binaries among pulsars characterized by short spin periods and low magnetic fields, such that binarity seems to be a characteristic of their formation and birth properties (e.g., see Verbunt 1990). High mass binary pulsars (HMBPs) are believed to originate from massive binary systems; during the violent formation epoch some combinations of recoil velocities and masses can leave two neutron stars, of comparable mass, bound in a relative orbit. An analysis of orbital parameters on the basis of statistical mechanics and energy minimization should be conducted to determine the expected eccentricity; further, on the basis of the same characteristics, the orbital evolution should be studied. This will throw light on the formation of such systems. Observations indicate younger HMBPs (e.g., B2303+46 with an age $\sim 3 \times 10^{7}$ yr) have very eccentric orbits $(e \approx 0.7$; Thorsett et al. 1993), while older ones (e.g., B1543+12 with an age $\left.\sim 2 \times 10^{8} \mathrm{yr}\right)$ have less eccentric orbits $(e \approx 0.3$; Wolszczan 1991). This is indicative of a circularization process in progress to minimize the energy of the system.

\section{Orbital Dynamics and Distribution of Eccentricity}

Chatterjee \& Magalinsky (2000) consider the problem of the dynamics of two compact bodies (which in this context can be considered as a binary pulsar) with two degrees of freedom, corresponding to their separation and effective size using a condition which favors energy minimization and implies gravity softening. On the basis of statistical mechanics, considering the bound binary to be a subsystem in a microcanonical ensemble, we determine the equilibrium distribution of system parameters. The distribution of the eccentricity, for a given energy, has the form

$$
W\left(\epsilon ; \epsilon_{m}\right)=Q^{-1} \epsilon^{2}\left(1-\epsilon^{2}\right)^{-1}\left(\epsilon_{m}^{2}-\epsilon^{2}\right)^{-1 / 2},
$$

with $Q=(\pi / 2)\left[\left(1-\epsilon_{m}^{2}\right)^{-1 / 2}-1\right], \epsilon$ being the eccentricity and $\epsilon_{m}$ its maximum value for a given energy. Thus the equilibrium distribution function is a monotonically increasing function of eccentricity, limited by its maximum value for a given energy. This is such that (after the initial violent phase) binary pulsars are 
expected to have eccentric orbits. A point to be noted is that the distribution is almost insensitive to the orbital and spin angular momentum.

Analytical results on the basis of statistical mechanics (Magalinsky \& Chatterjee, in preparation) indicate that the orbit will circularize in the adiabatic regime on a large timescale; ultimately, circularization will take place in an effort to minimize the energy of the system (e.g., Lynden-Bell \& Pringle 1974).

\section{References}

Lynden-Bell, D., \& Pringle, J. E. 1974, MNRAS, 168, 603

Magalinsky, V. B., \& Chatterjee, T. K. 2000, Astron. Astrophys. Trans., 18, 807

Thorsett, S. E., Arzoumanian, Z., McKinnon, M. M., \& Taylor, J. H. 1993, ApJ, 405, L29

Verbunt, F. 1990, in Neutron Stars and their Birth Events, ed. W. Kundt, (Dordrecht: Kluwer), p. 179

Wolszczan, A., 1991, Nature, 350, 688 